\title{
A Sustainable knowledge repository for decision support in Climate Change Adaptation
}

\author{
Shah J. Miah, John G. Gammack \\ "Institute for Integrated and Intelligent Systems, \\ Griffith University, Brisbane, Australia \\ \{s.miah, j.gammack\}@griffith.edu.au
}

\begin{abstract}
Issues around climate change are being examined globally across various disciplines and informed decisions at community levels are required within wider national responses. Research addressing decision support for managing the possible impacts on human communities has to date been limited. As climate science knowledge and government policy changes, information must be updated for dissemination to regional implementers. Traditional knowledge repositories for decision support however work on static models, expensive to update in dynamic fields. This paper describes a process for developing a sustainable knowledge repository for decision support that can assist local policy development in climate change applications. An ontology guaranteeing the semantic integrity of the repository is a central feature. Using a semantic wiki approach the semantic integrity of the knowledge base is combined with the update of emergent findings from multiple stakeholders. Both methodological and practical aspects for developing an information framework to support climate change decision makers are addressed.
\end{abstract}

Keywords: knowledge repository, design science, sustainable solution, climate change, decision support

\section{INTRODUCTION}

Climate change is one of the most pressing problems of our time, and governments seek to implement effective policies based on the best emerging science. Documents, policies, new findings and trend projections are just some relevant aspects of knowledge that require to be collected, coded and integrated in knowledge repositories used by decision makers. Equally, local knowledge of regional factors, such as water, soil and microclimates moderate the interpretation of exotic studies as the knowledge becomes actively interpreted in local contexts. Knowledge repositories however are typically static, often based on fixed schemes, and containing policies and older science that itself is under continuous review. Moreover, in supporting decisions centralised findings cannot be expected to reflect the detailed knowledge of a particular region, and the possible impact of a blanket policy on a community. Pattern changes in agricultural crops, resulting from market or other pressures can over time contribute to environmental change. For example "rice growing in Australia diverts water away from use by natural ecosystems, but without adding much economic value to that water" [1]. Traditional knowledge and longer term awareness within affected regions can mitigate the impacts and risks of generic policies in this regard.

A new conceptual structure is required, that can combine the knowledge emerging globally with local factors to provide a sustainable solution that is regionally sensitive and practicable, whilst remaining principled, compliant to national policy, and fully up to date with emerging science and directives. This paper outlines the design of such a repository, after briefly detailing some relevant background to the climate change application.

Many researchers have identified issues that arise from climate change for both human/social systems and other ecosystems. Similarly researchers such as Bosch, Allen, Williams, Ensor [2] describe an integrated approach to assist manage rangelands in New Zealand, by combining local and scientific knowledge for decision making. Similar research on recycled water usage can be found in Mitakakis, Sinclair, and Leder [3]. These types of research helps maximise the community knowledge for enhancing their process rather than tracking the change in biological or ecological process due to the climate change, so that the policy makers can make correct decisions on adaptation policies. This research therefore intends to develop a process for decision support that can assist policy makers in building policies for climate change adaptation in specific zones or regional areas in other countries.

The paper aims to illustrate the role of semantic technologies in developing sustainable knowledge repositories for decision support. Following some background we outline issues in the climate change management domain and describe a novel repository design to address these. A novel approach of semantic 
wiki shows its integrity for personal knowledge management. This motivated us in outlining the proposed solution.

\section{BACKGROUND}

This section describes both theoretical and methodological background and focuses on methodological choices relevant to decision support and repository development.

\section{A. Theoretical background}

The main challenge is to outline a comprehensive process to track changing impacts on human or social systems due to climate change. This process will enable a single accessible, commonly understandable knowledge repository that can establish a standard together with local and scientific knowledge for developing new policy adaptations. Decision support systems (DSS) have long history of addressing this type of issue in different business domains (for example in credit analysis [4]. Traditional Decision Support Systems (DSS) however, have shown limited success in such a problem domain where problem situations are rapidly changing. There remain issues in decision process with user factors such as limited fit with the specific context of decision-making problems, differences in problem definitions between local users and researchers/solution developers, and completely being incapable to meet rapidly changing requirements [5]. The decision support processes for diagnosis often employ a complicated process of inspection and selection of symptoms within pre-set constraints. This approach is too rigid to cope with rapid changing situations. Some approaches use data-driven rather than goal-driven strategies to draw new conclusions from the existing situation. For example, Mansingh, Reichgelt and Bryson [6] describe an approach called CPEST which gives expert advice on the management of coffee farms, particularly in mountainous regions. This system provides the coffee farmers with expert knowledge on climate, topology and soil type of the farm, however, this approach may not be sustainable with given the need to adjust to the changing requirements of the regional agricultural practices, especially without any reengineering effort. Nonetheless, this type of approach can not track the change factors for further use. Likewise, Mahaman, Passam, Sideridis, and Yialouris [7] present a diagnostic advisory rule based system (DIARES-IPM) for integrated pest management in solanaceous crop systems. However, this system does not cope with climate issues that can be the cause of various pest problems. This type of system is typically limited in its ability to process quantitative knowledge for monitoring of day-to-day activities and does not undertake knowledge verification. Qin and Paling [8] discuss the requirements of common and controlled vocabulary in modelling problem domains that could be well accepted by all parties. As such it is important to have a basis such as a knowledge repository for application development that provides a common understanding on the problems specification that are reusable, sharable and interoperable.

We outline a process for developing the DSS as shown in figure 1 . With the involvement of local community and climate change scientists the entire process development activities compress with five components: knowledge acquisition for obtaining knowledge components from the problem domain; Prototype building for converting the problem knowledge into system components; Evaluation for justifying the solution building; Standardisation for conceptualising of database and solution model; and finally testing and verifications are for adjusting the developed process with the requirements of users, situations and processes.

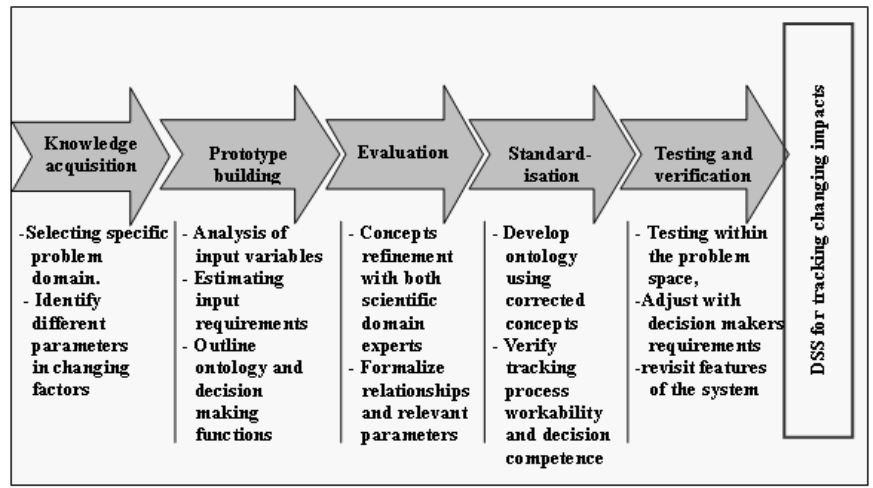

Figure 1: Ongoing research activities of the development of DSS

\section{B. Methodological background}

As mentioned the main aim of the project is to initiate a community-based development research approach for outlining a new decision support approach in which knowledge from multiple sources can be integrated in a common understandable repository for its further application development. From a societal viewpoint, solution developed in computer oriented discipline can be considered as a form of information systems whose main role is to mediate communication between parities [9]. Dini [9] describes computer science as "(being concerned) with the construction of abstract machines (and their) performance, self-optimisation, self-healing 
capacities" (p.27). This philosophical view motivates building or designing a system or solution artefact that can operate within objective or subjective reality within our social settings. From the social science point of view, there are four different philosophical paradigms that play a central role in guiding any information systems (IS) development process [10]. These paradigms' dimensions have been recognized within the IS research community since their conception. Hirschheim and Klein [10] mapped the four IS development paradigms, which were adapted from Burrell and Morgan's work [11]. These paradigms are: Functionalism, Social Relativism, Neohumanism and Radical Structuralism. However, the four IS development paradigms as ideal types, their properties are overstated to provide theoretical clarity: in practice overlap occurs and the differences are less extreme [10]. Such design activities could be improved if the problem situations and user requirements adequately reflected and mapped into software systems under methodological viewpoint. However, Ciborra and Hanseth [12] have argued that it is not easy to make a clear line between the objective view of the technology we build and our subjective human experience. This applies especially in our case where the decision-making problems lie inbetween the natural and human settings.

Recent works in design science suggest methodologies for information systems artefact design. Gregor and Jones [13] described the design science paradigm as a problem-solving oriented area that brings all inventions for artefact design together. It implies that design science research could help produce shareholderoriented and solution-relevant design through a rigorous and iterative process that includes evaluations with target users and communication of the solution to both operational and management users [14]. This view of technical design focuses more on specific design or a design from the individual point of view, rather than focusing on technical system design within sociotechnical phenomena such as a comprehensive design approach within social and natural settings. However, a digital ecosystem understanding goes beyond such a combined effort of developing a decision support framework as it embraces a diversity of community implications (i.e public health and environmental perspectives). The digital ecosystem promises more precisely to describe the interdependent view of technical system design within socio-technical phenomena, where we build or design a solution system. This approach could help track the changes in natural settings for enhancing effective communications within the community [9]. To guide the current project activity, according to the above discussion, we would be utilising participatory-based-development research under the digital business ecosystems paradigm.

\section{PROBLEM DOMAIN}

Climate change science is an interdisciplinary area that addresses diverse risk factors and potential impacts on human, social and environmental systems. Recent dramatic disasters such as earthquakes and tsunamis, and more insidious problems, such as desertification and rainfall imbalances require urgent and coordinated policy responses. Such responses must be both as informed, and as practicable, as possible. Because of the nature of ecosystems, imbalanced activity in one part has consequences for the whole system. Different specialist sciences regarding temperatures, water levels, wildfire risk, soil composition, salinity levels, and species habitat are all relevant considerations, and their interconnectedness implies direct impacts on sustainable human life and on the potential viability of local economies.

The science itself is being generated rapidly - policies and methods based on old science can have dangerous consequences: for example underestimating the water resource by measuring surface water rather than groundwater. The typical timings of national, regional and supranational policy cycles, and their implementation specifics however, are not suited to handling rapid changes as new knowledge becomes discovered, or when an unanticipated tipping point is reached, requiring policy adaptation. Such interventions require a much more adaptive response to evaluation than nominating a time horizon for political or bureaucratic reasons [15].

Apart from the underlying environmental science, policy is shaped by human interests, and what a community will accept in practice. Australia suffered several years of drought at the start of this century, and whilst Queensland successfully took restriction measures to get water consumption down, recycling sewage for drinking water still remains unpopular among an under-informed public [16]. Policy measures responding to climate change have impacts on society, and affect communities differentially if these are set centrally. Naturally local conditions will vary - and setting, (say) water targets must be done with regard to the needs of local industry, freshwater availability levels, purification or desalination capability and other factors that cannot be anticipated in detail at policy level. The local knowledge of these factors, and thus the relative importance for allocating resources and addressing 
specific impacts must also be taken into account. The education of the community, but also relevant inputs from those affected is required to achieve a holistic solution.

\section{ONTOLOGY BASED REPOSITORY}

We use the Protégé II knowledge environment to develop an ontology-based design. To date, ontology approaches have been used for building knowledge based systems by assembling reusable components. Knowledge engineering communities favour ontology methods because ontologies capture domain knowledge in a generic way and provide common, agreement-based understanding of a problem domain. This common basis enables reusable and sharing options across applications and users [17, 18].The proposed knowledge repository offers reconfigurable and updatable features both for policy makers and scientific domain experts. The first module is designed for knowledge acquisition in which a domain expert can acquire, formulate, and define the knowledge components. The problem formulation enables a process of adding new knowledge components into the knowledge repository. The knowledge components comprise different climate factors, and recognised impacts for building relationships between them. We now detail the repository design.

\section{Development of Knowledge Repository}

Many methodologies of knowledge modelling have been proposed for solution design. For instance, CommonKADS [19] is considered as the leading methodology to provide structured knowledge modelling and supports corporate-based knowledge management by including methods for analysing knowledge intensive tasks and processes. Abdullah, Benest, Evans and Kimble [20] noted that the knowledge model of CommonKADS incorporates application development processes through the use of a class diagram, a use case diagram, an activity diagram and a state diagram. CommonKADS provides tools for corporate knowledge management tasks and knowledge intensive processes rather than capturing domain knowledge and modelling the knowledge for application development [20, 21]. Task based modelling and requirements capture through use cases is the priority in this approach, which is in the classic tradition of structured systems analysis and development, evolved to be compatible with objectoriented development. Along with task and procedural information, expertise is modelled as a set of static declarative relationships between domain objects. Such an approach however, whilst adequate for static application domains is fundamentally unsuited to situations where requirements change, and the developed system's model of expertise becomes less relevant. CommonKADS also separates the expertise model from any implementation guidance, thus knowledge analysis and systems design are disjoint and a recognised source of software failure and lack of uptake.

Protégé was developed by the Knowledge Modelling Group at Stanford Medical Informatics [22] by using ontology-based approaches. The first version of Protégé was developed to simplify the knowledge acquisition process for domain specific knowledge. Protégé II included a design environment for building knowledgebased systems Gennari et al. [24], and more powerful problem solving methods. This approach uses an ontology editor for knowledge acquisition during development, and supports different ontology languages. Protégé has evolved towards web and wider application integration, and has the advantage that "software building blocks and conceptual building blocks can be identical" $[22,23]$.

Many researchers suggest that the semantic web technology has potential to provide enhanced management of web based information. Many critics however have equally suggested this to be impractical beyond narrow domains. The semantic web promises to bring an annotational rigour to allow informed searching and application linking but the reality since BernerLee's original proposal is that Web 2.0 applications, characterised by end-user design, semantics and emergent norms now dominate, and the semantic web will be part of web 3.0, if that is indeed the future. Just as commonKADS was born in an era of fixing detailed requirements in analysis for structured software engineering and has evolved towards object orientation, protégé was conceived before the web and end user computing, and is only now evolving to adapt to those trends. A newer approach, designed in the context of massive and current information availability is the semantic wiki, [25] which offers a new conceptual data structure e.g. for personal knowledge organisation [26]. Semantic wikis allow the rapid updating of information, whilst introducing some formality to the structure through semantic web concepts, such as an ontology of the wiki concepts [27] that allows some semi-automatic processing of content, as well as enhanced navigation and knowledge reuse [25]. 
Using the example of organising research knowledge about sharks into a repository, the conceptual data structure proposed by Volkel and Haller [26] allows stepwise formulation into a model to record, structure and communicate the knowledge at different levels of formalisation and granularity. A set of tools, including one resembling a semantic wiki, allows a range of knowledge inputs to be integrated, from paper notes and scans to bookmarks and structured documents. This type of approach allows ontologies to be built, refined, accepted and integrated with other applications by their user(s).

We believe this approach applies beyond personal knowledge management for organising knowledge in the climate change domain. Particularly when some of the core concepts are contested, or definitions at data dictionary level would represent unacceptable political commitments, negotiation and formalisation can be gradually modelled.

Although the ongoing negotiation mechanisms for refining emerging and evidenced understandings are specifiable through standard wiki functionality, we can at least identify a set of common knowledge components and those components can be converted into taxonomies in domain ontology immediately, ultimately allowing general querying and view building not available in normal wikis [25].

Table 1 shows how we convert the knowledge components into domain ontology for climate change. Terminology used is consistent with climate change literature, particularly from Gunasekera Kim, Tulloh and Ford [28] and McMichael et al. [29]. Figure 1 illustrates an example of the ontology repository for the problem domain.

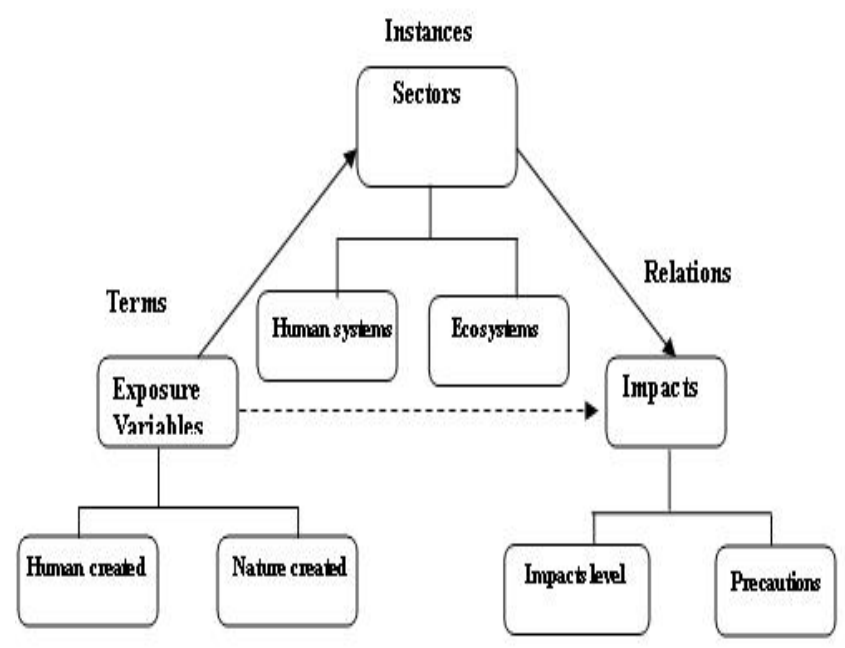

Figure 1: An example of a part of the ontology repository

\begin{tabular}{lll}
$\begin{array}{l}\text { Terms (i.e. Exposure } \\
\text { variables) }\end{array}$ & $\begin{array}{l}\text { Instances (i.e. } \\
\text { Sectors) }\end{array}$ & Relations (i.e. Impacts \\
Max temperature & Human health & Flood \\
Min temperature & Agricultural & Rainfall \\
Extreme rainfall & production & Crop suitability \\
Sea level & Natural disasters & Agricultural Pasts \\
Flood defences & Infrastructure & Water availability in invers \\
Carbon in air & Coastal and sea level & Cyclones \\
Vapour pressure & & Heat stress and discomfort \\
Mean temperature & & Vector-botne disease \\
& & Death rate \\
\hline
\end{tabular}

Table 1: Main components of the developed ontology based repository).

Exposure variables cause impact in different sectors. Exposure variables are those key variables, such as air temperature, that have a potential impact depending on their level in a given context, and may be naturally occurring or artificial. Sectors are modelled as instances that comprise sets of relations for defining the concepts in humanly understood and agreed terms. Specific instance scenarios would suggest a potential impact, and thus support the policy making of relevant decision makers. Such a methodology is common in climate change science, but this approach allows new knowledge 
and contextual information to be accommodated within the decision models built.

The proposed approach is comparable with a DSS development by Kim, Nute, Rauscher and Loftis [30] in which a reusable, domain-independent, and open-ended technique is used for developing DSS application. Their multi-component based approach integrated IS components such as simulation models, databases and specific applications like Carbon emission monitoring systems. Here we use an ontology based representation which is interoperable with existing systems and uses those to perform new reasoning (cf. Benjamins and Gomez Perez, [31]).

Figure 2 shows a conceptual model for a knowledge repository that can provide both scientific and contextual knowledge for policy making decisions.

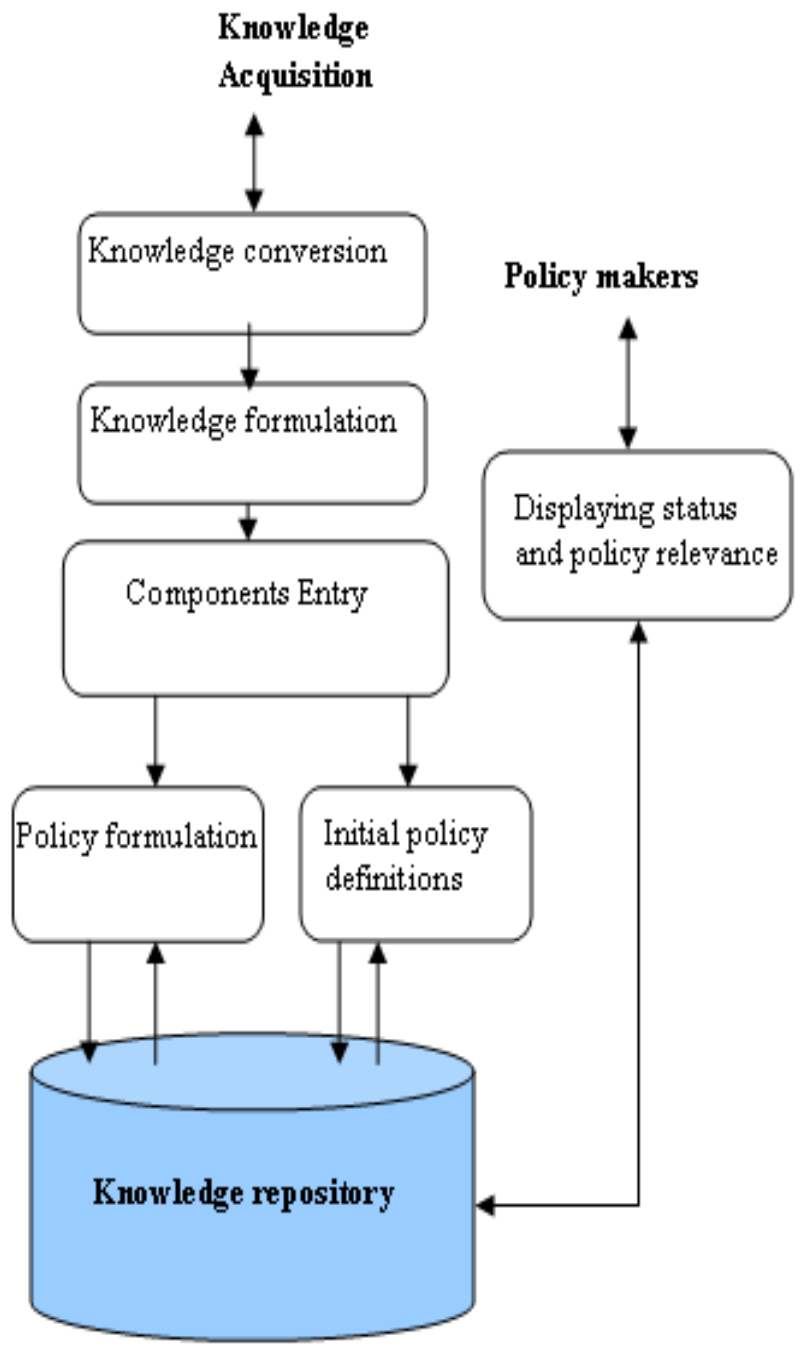

Figure 2: A conceptual model of the proposed knowledge repository

In the knowledge conversion phase- domain experts define the exposure variables, outcome (impact) variables and sectors potentially affected. These are referenced to the definitive sources at the time. In the knowledge formulation phase- the separated components (described and modelled as terms, instances and relations) will be acquired and input through the components entry module. These models may be reshaped by inputs from new stakeholders, emerging science, or periodic review and revision. The knowledge components then pass through a policy formulation process, specified in the agreed terms, and setting (for example) target levels of industrial water use. These inputs again are shaped by experts using research on sustainability levels, and political insights on realistic acceptabilities - necessarily shaped at more local levels. This fulfils the operational and administrative requirements for decision support, allowing initial policies to be created, but revised as situations change since the ontology described is inherently reusable).

Decision makers/stakeholders operating in local contexts can enter the current values of the variables to see the comparison between current and desired impacts using an automatically generated and scientifically valid policy with respect to the new situation. Current data on water replenishment and usage patterns (in this example), will allow decision making sensitive to local realities and changing climate, and allows targets to be changed or other mechanisms to control the resources impacted.

Questions can be asked such as "What will be the overall freshwater requirement given expected changes in climate and increased urbanisation in this region by 2015?" The combination of parameter settings informed by the model built from best science and local knowledge can project a scenario that suggests policy responses.

\section{DISCUSSION AND CONCLUSION}

Whilst there is much emerging research on climate change, specific research integrating the science with the political realities of policy adaptation has to date been limited. As a holistic and global problem the science may be shared, but the political implementations require a local sensibility. A major challenge for society is to 
track changing impacts on human or social systems due to climate change.

Definitional agreement is essential if policy is to be coherently understood and implemented, implying shared measurement and modelling methodologies: there is a clear need for new systems to address this, along with integrating new scientific and political information coherently. This paper discussed a new systematic approach developed in relation to address these issues, incorporating an ontology based knowledge repository that is sustainable, up to date and locally relevant. The development process enables a single accessible, interoperable and reusable knowledge repository, which supports local policy adaptations but is also commonly understandable.

The next phase of this research is fully to develop and test the sustainability of such a knowledge repository, particularly in supporting decisions around the urgent problem of climate change and its management.

\section{REFERENCES}

[1] Urban Ecology Australia (2006) Water for Rice http://www.urbanecology.org.au/topics/waterforrice.html

[2] O.J.H. Bosch, W.J. Allen, J.M. Williams, and A.H. Ensor (1996). "An integrated approach for maximing local and scientific knowledge for land management decision-making in the New Zealand high country", The Rangeland Journal, 18 (1), (1996), 23-32

[3] T. Z. Mitakakis, M.I. Sinclair, and K. Leder. "Alternative water sources and reuse: what are the public health issues", Medical Journal of Australia, 180 (6) (2004) 260-261

[4] Yi Peng, Gang Kou, Yong Shi, Zhengxin Chen, "A Multicriteria Convex Quadratic Programming model for credit data analysis", Decision Support Systems, 44 (4), (2008), 1016-1030

[5] S.J. Miah, D. Kerr, J. Gammack, and R.T. Cowan. "A Generic Design environment for the Rural Industry Knowledge Acquisition", Knowledge Based Systems Journal, 21, (2008) 892-899

[6] G. Mansingh, H. Reichgelt and K. O. Bryson. "CPEST: An expert system for the management of pests and diseases in the Jamaican coffee industry". Expert systems with Applications, 32 (2007) 184-192.

[7] B. D. Mahaman, H. C. Passam, A. B. Sideridis, and C. P. Yialouris. "DIARES-IPM: a diagnostic advisory rule - based expert system for integrated pest management in Solanaceous crop systems", Agricultural Systems, 76 (2003) 1119-1135.

[8] J. Qin and S. Paling. "Converting a controlled vocabulary into an ontology: the case of GEM", Information Research, 6(2) (2001) (URL: http://InformationR.net/ir/6-2/paper94.html

[9] P. Dini. "A scientific Foundation for Digital Ecosystems", Digital Business Ecosystems, European Commission, Luxembourg (2008).

[10] R. Hirschheim and H. K. Klein."Four paradigms of information systems development", CACM, 32(1989) 1199-1216.

[11] G. Burrell and G. Morgan. "Sociological Paradigms and Organisational Analysis", Heineman, London. 1979
[12] C. Ciborra, and O. Hanseth. Fron tool to Gestell: Agendas for managing the information infrastructure, Information Technology \& People (1998).

[13] S. Gregor and D. Jones. "The Anatomy of a Design Theory", Journal Assoc. for Information Systems (8:5), (2007) 312.

[14] A.R. Hevner, S.T. March, J. Park and S. Ram. "Design Science in information systems research", MIS Quarterly, 28(1) (2004) 75.

[15] M. Hildén, Time horizons in evaluating environmental policies. In M. Birnbaum \& P. Mickwitz (Eds.), Environmental program and policy evaluation: Addressing methodological challenges. New Directions for Evaluation, 122, (2009). 9-18.

[16] P. Brown. Tipping Point, Red, Issue 3, May, (2009) Griffith University, Australia.

[17] B. Chandrasekaran, J. R. Josephson, and V. R. Benjamins, Ontologies: What are they? Why do we need them? < IEEE Intelligent Systems and Their Applications, 14 (1) (1999) 20 26

[18] M. Blázquez, M. Fernández, J. M. García-Pinar, A .GómezPérez, A. "Building Ontologies at the Knowledge Level using the Ontology Design Environment", The 11th Knowledge Acquisition 164 Workshop, Banff, Alberta, Canada (1998).

[19] G. Schreiber, H. Akkermans, A. Anjewierden, R. de Hoog, N. Shadbolt, W.V de Velde, and B. Wielinga, Knowledge Engineering and Management: The CommonKADS Methodology, (1999). MIT Press, Massachusetts.

[20] M.S. Abdullah, I. Benest, A. Evans, C. and Kimble, C "Knowledge Modelling Techniques for Developing Knowledge Management Systems", 3rd European Conference on Knowledge Management, Dublin, Ireland: 15-25 (2002). (URL: http://www.cs.york.ac.uk/mis/docs/ECKM2002.pdf Accessed on 11th April, 2008).

[21] A. Manjarres, S. Pickin, and J. Mira, Knowledge model reuse: therapy decision through specialisation of a generic decision model, Expert Systems with Applications, 23, Issue 2, August (2002), 113-135,

[22] M. A. Musen. Automated Generation of Model-Based Knowledge-Acquisition Tools, Pitman Publishing, (1989) (URL: http://portal.acm.org/citation.cfm?id=73929, Accessed on January 20,2008).

[23] M.A. Musen, State of Protégé Keynote, $11^{\text {th }}$ protégé conference, Amsterdam (2009) (URL: http://protege.stanford.edu/conference/2009/slides/Musen_Prot ege_No\%20longer\%20getting\%20carded.pdf)

[24] J.H. Gennari, M.A. Musen, R.W. Fergerson, W.E. Grosso, M. Crubezy, H. Eriksson, N.F. Noy, S.W. Tu . The evaluation of Protégé: An Environment for Knowledge Based systems development, International Journal of Human-Computer Studies, 58 (1), (2003), 89 - 123. (URL: http://smiweb.stanford.edu/pubs/abstracts_by_author/Noy,N.papers.html, Accessed August, 7, 2008).

[25] E. Oren, Semantic Wikis for Knowledge Workers CIMRU/DERI/HP Seminar, Galway, (2005), (URL: http://www.m3pe.org/seminar/oren.pdf )

[26] M. Volkel and H. Haller, "Conceptual Data Structures for Personal Knowledge management, Online Information Review, 33 (2), (2009), 298- 3125

[27] M. Buffa, F. Gandon, G. Ereteo, P. Sander, C. Faron, SweetWiki: A semantic wiki, Web Semantics: Science, Services and Agents on the World Wide Web, Volume 6, Issue 1, Semantic Web and Web 2.0, (2008), 84-97, ISSN 1570-8268, (URL: http://www.sciencedirect.com/science/article/B758F4R53WD4-4/2/e25e28ff5d03166ed76dbbcd6e7fcbdf) 
[28] D. Gunasekera, Y. Kim, C. Tulloh, and M. Ford 'Climate change: impacts on Australian agriculture', Australian Commodities, vol. 14, no. 4, December quarter, (2007), 657676

[29] A. Mcmichael, R. Woodruff, P. Whetton K. Hennessy, N. Nicholls, S. Hales, A. Woodward, and T. Kjellstrom, Human Health And Climate Change In Oceania: A Risk Assessment, (2002), Government Report, Commonwealth Of Australia.

[30] G. Kim, D. Nute, H. M. Rauscher, D. L. Loftis. AppBuilder for DSSTools: an application development environment for developing decision support systems in Prolog, Computers and Electronics in Agriculture, 27 (2000) 107-125.

[31] R. Benjamins, and A. Gomez-Perez, Knowledge-Systems technology: Ontologies and Problem-Solving Methods, (2000), (URL: http://www.swi.psy.uva.nl/usr/richard/pdf/kais.pdf. ) 